\title{
Photoionized plasmas induced in molecular gases by extreme ultraviolet and X-ray pulses
}

\author{
Andrzej Bartnik', Wojciech Skrzeczanowski, Henryk Fiedorowicz, Przemysław Wachulak, Tomasz Fok, Eukasz \\ Węgrzyński, Roman Jarocki
}

Institute of Optoelectronics, Military University of Technology, Kaliskiego 2, 00-908 Warsaw, Poland

\begin{abstract}
In this work a laser-produced plasma (LPP) source was used to create low temperature plasmas. An extreme ultraviolet and soft X-ray (EUV/SXR) radiation pulse was used for ionization of molecular gases, injected into a vacuum chamber synchronously with the EUV/SXR pulse. Energies of photons exceeding $100 \mathrm{eV}$ were sufficient for dissociative ionization, ionization of atoms or even ions. The resulting photoelectrons had also enough energy for further ionizations or excitations. Time resolved UV/VIS spectra, corresponding to single charged ions, molecules and molecular ions, were recorded. For spectral lines, corresponding to radiative transitions in F II and S II ions, electron temperature was calculated based on a Boltzmann plot method. Numerical simulations of the molecular spectra were fitted to the experimental spectra allowing for determination of vibrational and rotational temperatures.
\end{abstract}

\section{Introduction}

Low temperature plasmas are widely used in different technologies [1]. Probably the most important is application of plasma processes in the microelectronics industry. For the microfabrication of integrated circuits, several fabrication steps are typically plasma based [2]. The other very important application is deposition of thin films $[3,4]$. This type of deposition allows for production of layers having unique properties like hydrogenated silicon layers or diamond-like carbon. Another important process is plasma etching $[5,6]$. Reactive etching is connected with formation of volatile products like $\mathrm{FW}_{6}$, $\mathrm{GeF}_{4}$ or $\mathrm{SiF}_{4}$. This effect can be thus utilized for reactive plasma etching of tungsten, germanium, silicon or other materials containing these elements like $\mathrm{SiC}$ [7] or glass [8]. Plasma etching is widely used for manufacturing of micro-electromechanical systems (MEMS), microfluidic devices, optical diffractive components, X-ray diffractive or refractive optics or in different kinds of nanotechnologies. Plasma treatment can be also employed for surface activation and functionalization of polymers [9]. Careful and appropriate selection of the types of plasma source could introduce various functional groups on polymer surface in order to change wettability, adhesion characteristics, and biocompatibility of polymers by directly changing the surface chemical compositions [10-12]. Crosslinking of the polymer chains results in increasing of the molecular weight of polymers and could cause polymer surface to establish three-dimensional molecular networks, by which the polymer layer became an insoluble material [13]. Fluorination of polymers by plasma treatment results in hydrophobic surfaces without degradation of their bulk properties $[14,15]$. The $\mathrm{SF}_{6}$ plasma treatment of silicon surface can convert it to so called black silicon with minimized reflection losses. Low reflectance of the black silicon is very important for optoelectronic devices, in particular, for high efficiency solar cells [16]. Fluorination was also employed to improve properties of amorphous carbon films resulting in increase of the film roughness and hydrophobicity [17]. Apart from that, SF6 plasma treatment can be employed for functionalization of graphene and carbon nanotubes $[18,19]$. Low temperature plasmas are also utilized for spectrochemical analysis. In particular it was shown by various authors that the excitation of atomic or molecular species in cold plasmas is a powerful method for such analysis. The method was used for a large variety of analytical tasks such as the determination of noble metals [20], the determination of the rare earth elements [21] or the determination of $\mathrm{Hg}$ with the aid of continuous cold vapour generation [22]. In this case the sample aerosol was introduced into the low temperature plasma and the emission spectra of the excited species were measured and analysed.

For creation of plasmas used in these technologies plasma generators based on electrical discharge, working in a stationary regime, are used. They allow to create plasmas of low temperature $\left(\mathrm{T}_{\mathrm{e}} \sim 10^{4} \mathrm{~K}\right)$ and low electron density $\left(\mathrm{n}_{\mathrm{e}} \sim 10^{11}-10^{13} \mathrm{~cm}^{-3}\right)$ [23]. Even in plasmas produced under atmospheric pressure the electron density, hence, also ion density $\left(\mathrm{n}_{\mathrm{i}}\right)$ does not exceed these values. In our previous works, an alternative method of low temperature plasmas creation, by irradiation of gases with extreme ultraviolet (EUV) or soft X-ray (SXR) pulses, was proposed. A possibility to create plasmas of significantly higher electron density,

*Corresponding author: andrzej.bartnik@wat.edu.pl 
comparing to plasmas produced using standard generators, even exceeding $10^{18} \mathrm{~cm}^{-3}$ [24], was demonstrated. All these works concerned spectral investigations of atomic or ionic species. In this work EUV induced plasmas, created by irradiation of molecular gases, were investigated. Time resolved spectral measurements in the optical range were performed. Based on ionic and molecular spectra, the electron, vibrational and rotational temperatures were estimated.

\section{Experimental arrangement}

In our experiments, a $10-\mathrm{Hz}$ laser produced plasma (LPP) EUV/SXR source, based on a double-stream gaspuff target, irradiated with the $10 \mathrm{~ns} / 5 \mathrm{~J} \mathrm{Nd:YAG} \mathrm{laser}$ pulses, was used. The target was formed by pulsed injection of xenon gas into a hollow stream of helium by employing an electromagnetic valve system, equipped with a double nozzle set-up. The laser focusing conditions and plasma parameters were adjusted such as to obtain maximum emission in the EUV spectral region. The most intense emission was in the relatively narrow spectral region centred at $\lambda \sim 11 \pm 1 \mathrm{~nm}$, however, significant part of the radiation was emitted in a broad spectral range $\lambda \sim 1-20 \mathrm{~nm}$. The LPP source was used to ionize a small portion of gas injected into the vicinity of the laser plasma. The gas was injected using an auxiliary gas puff valve synchronized with laser pulses. Its density in the interaction region could be regulated within the range of the order of $1 \div 10 \%$ of the atmospheric density. Ionization of the gas resulted in creation of low temperature plasmas. Spectral investigation of these plasmas were performed in a UV/VIS range $(\lambda=200$ $780 \mathrm{~nm}$ ), using an Echelle Spectra Analyzer ESA 4000. To avoid a strong radiation from the laser plasma, it was screened by a specially prepared metallic plate mounted between the plasma and the spectrograph. Apart from that the measurements were performed with a temporal resolution which allowed to register exclusively spectral lines originating from the EUV induced plasmas, and not from the expanding laser plasmas.

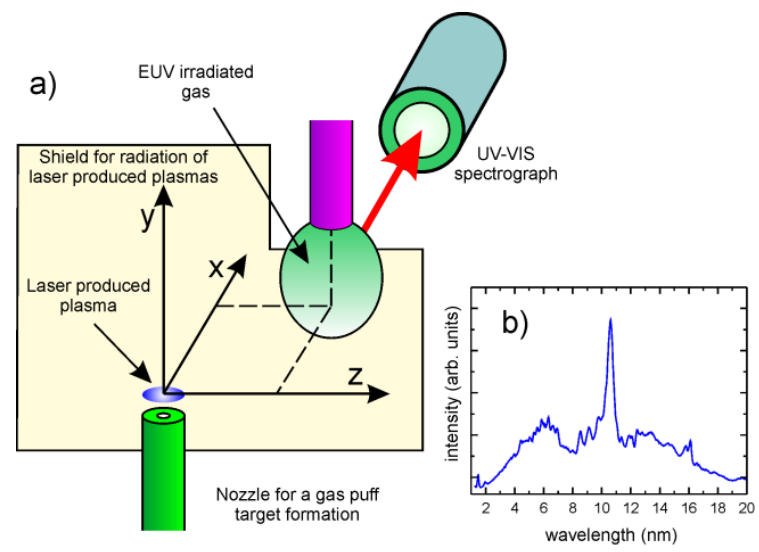

Fig. 1. Creation of EUV induced low temperature plasmas: a) experimental arrangement, b) spectrum of the LPP radiation pulses
A schematic view of the experimental set-up together with the spectrum of the LPP source is presented in Fig.1.

\section{Experimental results}

Irradiation of molecular gases, injected into the interaction region, by EUV/SXR photons resulted in numerous processes including dissociative ionization or creation of molecular ions. Photoelectrons released during these processes have sufficient energies for further ionization or excitation of atoms, ions and molecules. This work is devoted to spectral investigation of low temperature plasmas induced in a nitrogen and SF6 gas. Spectra were acquired for different delays according to the ionizing pulse. The most intense spectrum, recorded at a time delay $t=150 \mathrm{~ns}$ after the gas irradiation by the EUV/SXR pulse, contains series of lines corresponding to vibrational emission bands of $\mathrm{N}_{2}$ and $N_{2}^{+}$species. In Fig. 2 two parts of the $N_{2}, C^{3} \Pi_{u}-$ $B^{3} \Pi_{g}$ electronic system, recorded in the UV range, are presented.
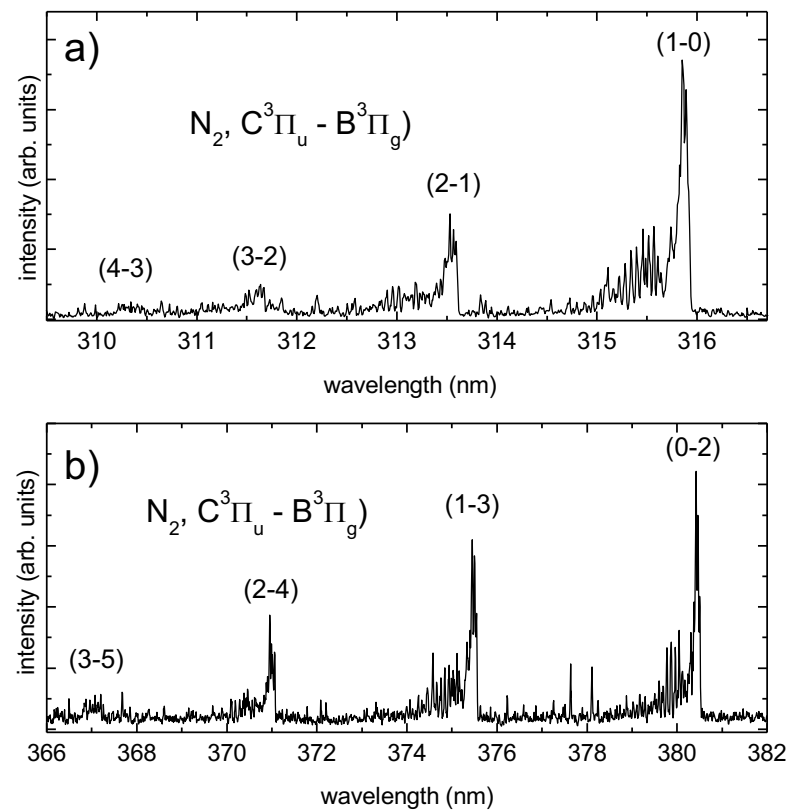

Fig. 2. Vibrational-rotational $N_{2}$ spectra corresponding to the $C^{3} \Pi_{u}-B^{3} \Pi_{g}$ electronic system: a) $\Delta v=-1$, b) $\Delta v=2$

Due to sufficiently high resolution of the spectrometer, $\lambda / \Delta \lambda \approx 20000$, not only the vibrational but also rotational structure of the spectra corresponding to the transitions within the $C^{3} \Pi_{u}-B^{3} \Pi_{g}$ system, are visible. The band heads were identified based on the Ref. 25 . Several other heads with the most intense one corresponding to the $(0-0)$ transition at the wavelength $\lambda$ $=337.1 \mathrm{~nm}$ were also detected.

As mentioned above, the spectrum contains also lines corresponding to transitions in the molecular ions $\mathrm{N}_{2}^{+}$. Their spectral intensity is similar or even higher comparing to the $N_{2}$ lines. Several vibrational emission bands of the $B^{2} \Sigma_{u}^{+}-X^{2} \Sigma_{g}^{+}$electronic system with a change of the vibrational quantum number $\Delta \mathrm{v}=$ 
$-1,0,1$, were recorded. Vibrational bands with $\Delta \mathrm{v}=$ -1 of the $B^{2} \Sigma_{u}^{+}-X^{2} \Sigma_{g}^{+}$electronic system and bands with $\Delta \mathrm{v}=1$ of the $C^{3} \Pi_{u}-B^{3} \Pi_{g}$ system are partially overlapping, hence, not very convenient for analysis. On the other hand, two other bands of the $B^{2} \Sigma_{u}^{+}-X^{2} \Sigma_{g}^{+}$ system, with $\Delta v=0,1$ are well separated from the $N_{2}$ spectrum. Hence, this part of the $N_{2}^{+}$spectrum could be used for estimation of the vibrational and rotational temperatures. The estimation was based on simulation of the spectrum employing the LIFBASE code [26]. Simulations were performed for several vibrational and rotational temperatures ( $T_{v}$ and $T_{r}$ respectively). The best fit was obtained for $T_{v}=3000 \mathrm{~K}$ and $T_{r}=200 \mathrm{~K}$. The corresponding experimental and calculated spectra are presented in Fig.3.
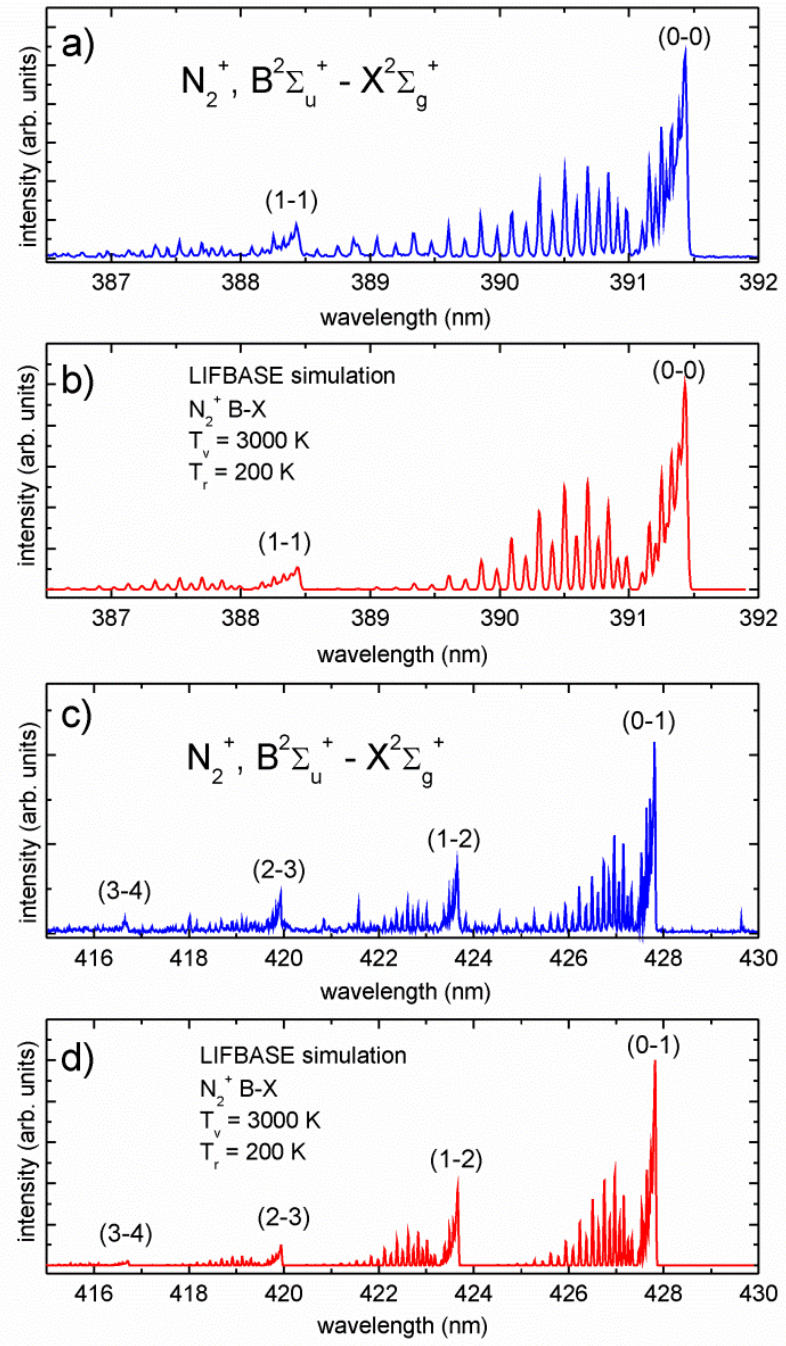

Fig. 3. Vibrational-rotational $N_{2}^{+}$spectra corresponding to the $B^{2} \Sigma_{u}^{+}-X^{2} \Sigma_{g}^{+}$electronic system: a) experimental, $\Delta \mathrm{v}=0$;

b) $\Delta v=0$, numerical simulation ; c) experimental, $\Delta v=1$;

d) $\Delta \mathrm{v}=1$, numerical simulation

Spectra recorded for different experimental conditions were dominated by the lines corresponding to the $N_{2}$ and $\mathrm{N}_{2}^{+}$molecular bands. Lines corresponding to radiative transitions in nitrogen atoms were not detected. On the other hand two spectral lines corresponding to singly charged ions $2 s^{2} 2 p 3 s\left({ }^{1} P_{1}^{o}\right)-2 s^{2} 2 p 3 p\left({ }^{1} D_{2}\right)$ and $2 s^{2} 2 p 3 s\left({ }^{3} P_{2}^{o}\right)-2 s^{2} 2 p 3 p\left({ }^{3} P_{2}\right)$ at the wavelengths $\lambda=$ $399.5 \mathrm{~nm}, \lambda=463.05 \mathrm{~nm}$ respectively, were revealed. Their intensities, however, are an order of magnitude lower comparing to the most intense molecular lines. According to the NIST database [27] these lines are the most intense among the N I and N II lines. It can be thus concluded that the other lines, including the $\mathrm{N}$ I lines, were too weak to be detected. It means that except the molecular ionization and excitation, the dissociative ionization or dissociation with further ionization of the nitrogen molecules also took place. It should be expected due to high energy of the driving photons, capable even for the inner-shell ionization.

In principle, based on the intensity ratio of two N II lines it would be possible to calculate the plasma electron temperature. On the other hand intensities of the lines can be affected by self-absorption, especially in case of the strongest lines. It means that an error of the temperature estimation, performed this way, could be significant. Much better is to utilize a Boltzmann plot method [28,29] based on relative intensities of multiple lines corresponding to radiative transitions between the levels $i$ and $k$. The plot is constructed from individual points with the values of $\ln \left(I_{k i} \lambda_{k i} / A_{k i} g_{k}\right)$ versus the upper level energy $E_{k}$. Quantities $I_{k i}, \lambda_{k i}, A_{k i}, g_{k}$ are as follows: the line intensity, its wavelength, the radiative transition probability and the statistical weight of the upper level, respectively. In case of LTE (local thermodynamic equilibrium) or at least partial LTE (pLTE) and spectral lines originating from the same ion species a straight line can be fitted to the points and the slope $1 / k T$ yields the electron temperature. The method could not be employed for the nitrogen plasma, due to lack of multiple $\mathrm{N}$ I or $\mathrm{N}$ II lines, hence, the measurements were performed for the plasma induced in other molecular gas, namely $S F_{6}$. In this case numerous lines originating from $\mathrm{F}$ II and $\mathrm{S}$ II ions were detected. In Fig.4 Boltzmann plots constructed for these lines are presented. The electron temperature estimated from these plots is of approximately $T_{e} \sim 2$. $10^{4} \mathrm{~K}$.

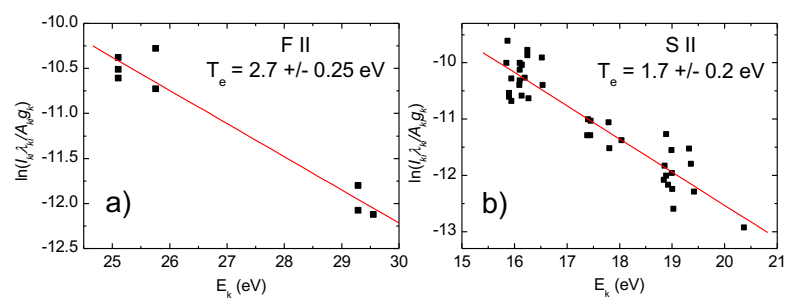

Fig. 4. Boltzmann plots constructed for spectra of single charged ions: a) F II, b) S II

Similar values were obtained in our earlier experiments with plasmas induced in rare gases [30,31]. It can be thus assumed that also for the nitrogen plasma the electron temperature is of the same order. In this case the electron temperature is almost an order of magnitude higher comparing to the vibrational temperature.

In Fig. 5 a temporal dependence of relative intensities of the (0-0) emission bands corresponding to the $C^{3} \Pi_{u}-$ $B^{3} \Pi_{g}$ and $B^{2} \Sigma_{u}^{+}-X^{2} \Sigma_{g}^{+}$systems are presented. At a 
sub-image a time profile of a typical EUV/SXR pulse, used for creation of the low temperature plasmas, was added. An FWHM (full width at half maximum) of the EUV pulse, measured using a fast AXUV HS1 detector, is $\tau=11 \mathrm{~ns}$. This value is comparable to the time duration of the driving laser pulse.

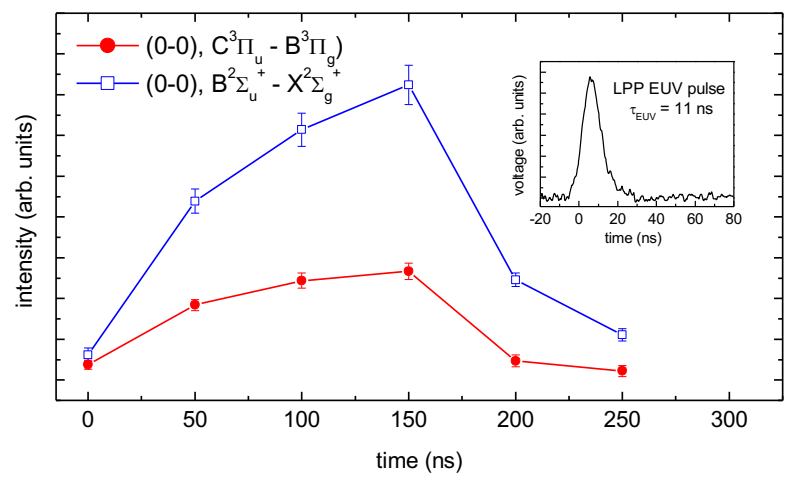

Fig. 5. Temporal dependence of relative intensities of emission bands corresponding to $N_{2}$ and $N_{2}^{+}$molecules. Time profile of the driving LPP EUV pulse at the sub-image.

As can be noticed from Fig.5 a time of the optical emission from the nitrogen plasma is much longer, over an order of magnitude longer comparing to the driving pulse. Apart from that, intensity of the UV-VIS radiation emitted from the plasma increases within a time of approximately $t_{N_{2}} \sim 150 \mathrm{~ns}$. It means that the molecular electronic excitations are driven mostly by collisional processes following the photoionization phase. Similar temporal dependence, presented in Fig. 6 was obtained for the emission line corresponding to the $2 s^{2} 2 p 3 s\left({ }^{1} P_{1}^{o}\right)-2 s^{2} 2 p 3 p\left({ }^{1} D_{2}\right)$ transitions.

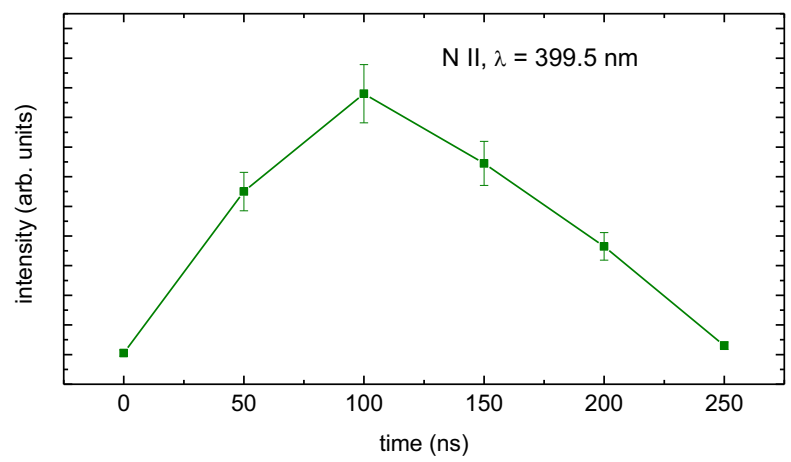

Fig. 6. Temporal dependence of relative intensities of the $2 s^{2} 2 p 3 s\left({ }^{1} P_{1}^{o}\right)-2 s^{2} 2 p 3 p\left({ }^{1} D_{2}\right)$ emission line

In this case, however, the intensity maximum is for the time $t_{N I I} \sim 100 \mathrm{~ns}$. It is reasonable taking into account that the dissociative ionization potential of the nitrogen is relatively high, $I_{N I} \sim 24.3 \mathrm{eV}$ and an additional energy exceeding $21 \mathrm{eV}$ has to be delivered for creation of the $2 s^{2} 2 p 3 p\left({ }^{1} D_{2}\right)$ excited state. These processes require a presence of electrons with relatively high energy, an order of magnitude exceeding the average electron energy corresponding to the electron temperature $\left(T_{e} \sim 2 \mathrm{eV}\right)$. Number of such electrons initially increases as a result of photoionization, Auger emission and further collisional ionization but after a finite time decreases, due to thermalization and non elastic collisions with atoms or molecules. From the temporal dependence in Fig.6 this time can be estimated to approximately $100 \mathrm{~ns}$.

\section{Summary}

In this work first results of investigations of low temperature plasmas produced by irradiation of molecular gases with intense EUV/SXR ionizing pulses, are presented. The gases were delivered into the interaction region synchronously with the ionizing pulses. The resulting low temperature plasmas were investigated using the UV/VIS spectroscopy. Emission lines corresponding to excited states in singly charged ions, molecular ions and neutral molecules were detected. Numerical simulations of the molecular spectra allowed to determine vibrational and rotational temperature of the nitrogen plasma. For spectral lines corresponding to radiative transitions in F II and S II ions, the electron temperature was calculated, based on the corresponding Boltzmann plots. Additionally temporal evolution of the emission from ionic and molecular species was presented. It was shown that the lifetime of the EUV induced plasma is an order of magnitude longer comparing to the time duration of the driving pulse.

This work was supported by the National Science Centre, Poland, grant agreement no. UMO-2016/23/B/ST7/00949, and partially by European Union's Horizon 2020 Programme (LASERLAB-EUROPE) grant agreement no. 654148

\section{References}

1. A. Bogaerts, E. Neyts, R. Gijbels, J. van der Mullen, Spectrochimica Acta Part B 57, 609-658, (2002)

2. M.A. Lieberman, Advanced Technologies Based on Wave and Beam Generated Plasmas, NATO Science 67, 1-22, (1999),

3. M. Kumar, Y. Ando, JNN 10, 3739-3758 (2010)

4. M. Konuma, Film Deposition by Plasma Techniques, Springer, New York, 1992

5. D.M. Manos, D.L. Flamm, Plasma Etching: An Introduction, Academic Press, New York, 1989

6. S. Samukawa, T. Mieno, Plasma Sources Sci. Technol. 5, 132-138, (1996)

7. N.O.V Plank, M.A. Blauw, E.W.J.M. van der Drift, R. Cheung, J. Phys. D 36, 482-487, (2003)

8. L. Lallement, C. Gosse, C. Cardinaud, M.-C. Peignon-Fernandez, A. Rhallabi, Journal of Vacuum Science \& Technology A 28, 277 (2010)

9. S. Yoshida, K. Hagiwara, T. Hasebe, A. Hotta, Surface \& Coatings Technology 233, 99-107 (2013)

10. K.R. Kull, M.L. Steen, E.R. Fisher, J. Membr. Sci. 246, 203, (2005) 
11. J.N. Lai, B. Sunderland, J.M. Xue, S. Yan, W.J. Zhao, M. Folkard, B.D. Michael, Y.G. Wang, Appl. Surf. Sci. 252, 3375, (2006)

12. D. Hegemann, H. Brunner, C. Oehr, Nucl. Instrum. Methods Phys. Res. B Beam Interact. Mater. Atoms 208, 281, (2003)

13. S. Tajima, K. Komvopoulos, Appl. Phys. Lett. 89, 124102 (2006)

14. E.C. Rangel, W.C.A. Bento, M. E. Kayama, W.H. Schreiner, N.C. Cruz, Surf. Interface Anal. 35, 179-183 (2003)

15. R.Y. Korotkov, T. Goff, P. Ricou, Surface \& Coatings Technology 201, 7207-7215 (2007)

16. Y. Xia, B. Liu, S. Zhong, C. Li, Journal of Electron Spectroscopy and Related Phenomena 184, 589592 (2012)

17. N.M.S. Marins, R.P. Mota, R.Y. Honda, P.A.P. Nascente, M.E. Kayama, K.G. Kostov, M.A. Algatti, N.C. Cruz, E.C. Rangel, Surface \& Coatings Technology 206, 640-645 (2011)

18. M. Baraket, S. G. Walton, E. H. Lock, J. T. Robinson, and F. K. Perkins, Applied Physics Letters 96, 231501 (2010)

19. A.J. Barlow and J.S. Quinton, ICONN 2010 Proceedings of the 2010 International Conference on Nanoscience and Nanotechnology, IEEE Xplore, 120-123 (2010)

20. F. Liang, D.X. Zhang, Y.H. Lei, H.Q. Zhang, Q.H. Jin, Microchem. J. 52, 181-187 (1995)

21. Y. Duan, Y. Li, X. Tian, H. Zhang, Q. Jin, Anal. Chim. Acta 295, 315-324 (1994)

22. J.F. Camun-Aguilar, R. Tereiro-Garcia, J.E. Sanchez-Uria, Spectrochim. Acta Part B 49, 475484 (1994)

23. K. Ogura, H. Yamada, Y. Sato, Y. Okamoto, Appl. Spectrosc., 51, 1496-1499 (1997)

24. A. Bartnik, P. Wachulak, T. Fok, H. Fiedorowicz, T. Pisarczyk, T. Chodukowski, Z. Kalinowska, R. Dudzak, J. Dostal, E. Krousky, J. Skala, J. Ullschmied, J. Hrebicek, T. Medrik, Physics of Plasmas 22, 4, 043302 (2015)

25. A. Lofthus, P.H. Krupenie, J. Phys. Chem. Ref. Data, 6, 113-307 (1977)

26. J. Luque and D.R. Crosley, SRI International Report MP 99-009 (1999)

27. http://physics.nist.gov/PhysRefData/ASD/lines_for m.html

28. C. Aragón, J.A. Aguilera, Spectrochimica Acta Part B 63, 893-916 (2008)

29. S. S. Harilal, Applied Optics, 43, 3931-3937 (2004)

30. A.Bartnik, P. Wachulak, H. Fiedorowicz, W. Skrzeczanowski, Physics of Plasmas 23, 043512 (2016)

31. A. Bartnik, W. Skrzeczanowski, P. Wachulak, I. Saber, H. Fiedorowicz, T. Fok, Ł.Węgrzyński, Laser and Particle Beams 35, 42-47 (2017) 